\title{
DISCURSOS SOBRE LA DISCRIMINACIÓN DE GÉNERO EN LOS TRABAJADORES MINEROS DEL NORTE DE CHILE
}

\author{
DISCOURSES OF GENDER DISCRIMINATION IN \\ MINING WORKERS IN THE NORTH OF CHILE
}

\section{PAULINA SALINAS MERUANE ${ }^{2}$ JAIME BARRIENTOS DELGADO ${ }^{3}$ PABLO ROJAS VARAS ${ }^{4}$}

\section{RESUMEN}

Este artículo tiene como objetivo analizar los discursos de género de los trabajadores mineros del norte de Chile referidos hacia las mujeres que trabajan en las "schoperías" (bares de cerveza) de Calama. Como premisa sostenida en evidencia previa, respecto a las relaciones de género en la zona, establecemos una relación estrecha entre el lenguaje de los hombres y las identidades masculinas dominantes que se recrean en este escenario productivo (minería). El habla actúa como una herramienta central en el reforzamiento de esta identidad. Por medio de una investigación cualitativa en las "schoperías" de la ciudad, se recopilaron los relatos de 23 trabajadores, obtenidos a través de 11 entrevistas en profundidad y dos grupos de discusión. Se identifican, a través de un sistema de codificación, distintas formas de expresar y encubrir la discriminación de género, en todas ellas, se menoscaba a las mujeres, se fortalecen relaciones de poder asimétricas y un sistema de sexo -género dominado por lo masculino.

Palabras clave: Discurso, género, discriminación, identidad masculina.

${ }^{1}$ El presente trabajo forma parte de los resultados del proyecto FONDECYT No 1070528 "Minería y relaciones de género: las transformaciones en el comportamiento sexual en la Región de Antofagasta, Chile".

${ }^{2}$ Doctora en Ciencias Sociales, Universidad Católica del Norte. Antofagasta, Chile. E-mail: psalinas@ucn.cl

${ }^{3}$ Doctor en Psicología Social, Universidad Católica del Norte. Antofagasta, Chile. E-mail: jbarrien@ucn.cl

${ }^{4}$ Magíster @ de Psicología Social, Universidad Católica del Norte. Antofagasta, Chile. E-mail: prlux2@yahoo.com 


\begin{abstract}
This article aims to analyze the gender discrimination discourse of male miners referred to women working in "schoperías" (beer bars) in Calama. As a premise supported by previous evidence with regard to gender relations in the area, we establish a close relationship between the language men use and the dominant male identities that are recreated in this productive scenario (mining). Discourse acts as central tool in the reinforcement of this type of identity. Through a qualitative research in the "schoperías", the accounts of 23 workers were collected, obtained through 11 individual in-depth interviews and two group discussions. Based on a coding system we identified different ways of expressing and concealing gender discrimination, in all of them women are undermined, reinforcing asymmetrical power relations and a sex-gender system dominated by masculinity.
\end{abstract}

Keywords: Discourse, gender, discrimination, masculine identity.

Recibido: 20.07.10. Aceptado: 11.01.11.

\title{
1. REVISIÓN DE LA LITERATURA
}

$\mathrm{E}$

XISTE UNA AMPLIA documentación sobre la construcción de las identidades de género, centrada en la situación de desigualdad que afecta a las mujeres (Valdés y Weinstein, 1993; Ruiz, y otros, 1998; Valdés, 2001; GIM, 2002; Lamas, 2002; Salinas 2004). En estas investigaciones se sostiene que la identidad femenina depende más de las cualidades sociales que biológicas (Fuller, 1998). Otros estudios, entre los que se hallan los aportes de Trautner (2005), añaden que las organizaciones igualmente están generizadas, es decir, contienen fuertes órdenes simbólicos de género que entregan claves a los sujetos sobre cómo comportarse adecuadamente en contextos sociales.

Particularmente, los estudios sobre masculinidad se inician en la década de los 80 y logran una presencia más significativa en los 90 . En ellos se abordan las relaciones de género en las sociedades latinoamericanas y las identidades masculinas. Actualmente, en América Latina hay investigaciones relevantes sobre la masculinidad (Careaga y Cruz, 2006; Olavarría, 2006; Seidler, 2006; Viveros, 2001; Valdés y Olavarría, 1997). Muchos de estos trabajos se basan en las experiencias vertidas en los estudios sobre mujeres, y han puesto atención en el carácter construido de la identidad. Connell $(2003,2006)$ plantea que los modelos de masculinidad cambian con el tiempo, en una misma sociedad, inclusive en una comunidad o institución (Valdés y Olavarría, 1997).

En el caso de Chile, específicamente en la Región Metropolitana, es fun- 
damental contextualizar la construcción de la identidad masculina, considerando las transformaciones socioculturales y económicas que ha vivido el país en las últimas décadas. Valdés y Olavarría (1997) han liderado los estudios en el país. Ellos afirman que no es posible conceptualizar una masculinidad única, sino más bien se trata de un proceso multifacético, a pesar del impacto que han tenido los modelos hegemónicos de masculinidad existentes y los efectos que tuvo la Dictadura Militar en la vida de los chilenos. Así, y tal como lo han señalado otros autores (Connell, 2003; Seidler, 2006), la identidad masculina se robustece en espacios de homosociabilidad, entre los que se destacan los bares, cafés, "schoperías" y night $c l u b$. En estos locales se privilegian estereotipos como: valentía, virilidad, fuerza sexual, poder económico, entre otros. Lo mismo se aprecia en los denominados butecos o bares, de acuerdo a lo que registra Jardim (1995) para el caso de Brasil.

Sin embargo, otros estudios documentan el actual proceso de transición que están experimentando los hombres en la construcción de su identidad, inclusive a nivel latinoamericano (Carabí y Armengol, 2008; Montecino, 2007, Valcuende y Blanco, 2003), lo que ha implicado un cuestionamiento de los estereotipos tradicionales asignados a hombres y mujeres. $Y$, aunque estas transformaciones no son generalizadas, dan cuenta de cambios en los imaginarios colectivos y marcan nuevas pautas de interacción en la vida cotidiana (Martínez, 2007).

Específicamente, en la minería hay una práctica fuertemente generizada; esto implica que la jerarquía social entre hombres y mujeres se encuentra legitimada y reforzada en la cultura organizacional. En estos espacios dominados por los hombres, sus privilegios se manifiestan a través de una diferenciación funcional del trabajo por género (Tallichet, 1995).

Las investigaciones históricas sobre la minería del norte de Chile han descrito el carácter masculino del movimiento obrero y sindical, los partidos políticos y la organización del trabajo (Aylwin et al., 1998; Salazar y Pinto, 1999; Zapata, 2002; Vergara, 2004; Carmagnani, 2006). También, investigaciones psicosociales analizan la situación de los hombres trabajadores, de las mujeres, las condiciones familiares, los efectos del sistema de turno y los roles que los sujetos desempeñan en la vida privada de los campamentos en las minas (Vergara, 2007; Montecino, 1998). Thomas Klubock (1995) en su estudio sobre el mineral El Teniente, en la zona centro sur del país, destaca la importancia de la ideología de género en el modelamiento de la clase trabajadora y cómo se estructuraron las identidades de la clase y de la comunidad que surgieron en el proceso de proletarización. Este autor afir- 
ma que son diversas las fuerzas que se combinaron para producir la masculinización del trabajo y la domesticación de las mujeres en la esfera privada.

En el norte del país, la minería es la actividad productiva más importante, no obstante, es un reducto que prevalece al margen del proceso social transformador de las identidades de género, en el sentido que se favorece la construcción de la identidad masculina dominante.

El trabajo es uno de los ámbitos centrales en la configuración de la identidad de los individuos, en la diferenciación entre los sexos, en la construcción de los géneros y en el establecimiento de las jerarquías sociales (Todaro et al., 2000). En la minería, el trabajo es un mecanismo de realización para los hombres, a través de éste forman un hogar, son proveedores y cumplen los roles socioculturales esperados para ellos (Ruitenbeek, 1967; Montesinos, 2002; Olavarría, 2000; Seidler, 2006). Los estudios coinciden en que los varones se encuentran exigidos por una dinámica en la que trabajo-dignidad y capacidad se relacionan en un círculo vicioso, que condiciona la idealización de la identidad masculina adulta (Ruitenbeek, 1967; Dunbar, 1994; Macheke y Campbell 1998; Salinas y Arancibia, 2006; Salinas, 2007). Las circunstancias laborales, los lugares de trabajo, las condiciones económicas y las estructuras de las organizaciones, influyen en la forma en cómo los hombres construyen la identidad, inclusive, a niveles muy íntimos, como la relación de pareja y la sexualidad (Connell, 2003). Así, la supremacía masculina se impone como resultado de una sumisión paradójica, ya que es consecuencia de lo que se conoce como violencia simbólica, aquella que es silenciada, amortiguada, insensible para sus propias víctimas, y que se ejerce a través de las estrategias simbólicas de la comunicación y del conocimiento. La dominación se ejerce en un sentimiento, en un estilo de vida, una manera de pensar, de hablar o comunicar (Bourdieu, 2007). Esta dominación desplegada por los hombres se manifiesta en la discriminación que se ejerce contra las mujeres, y se relaciona con el proceso de construcción de la identidad de ellos. Se expresa con una actitud activa, abierta o implícita, que la sociedad ayuda a controlar (Cortés, 2004).

En un estudio realizado por Cantero (2003) en la sierra de Huelva en España explica que, inclusive, el proceso de socialización que lleva a un niño a ser un hombre implica el aniquilamiento permanente de su femineidad; ser hombre significa cortar con el mundo de las mujeres y de la propia infancia, para acentuar únicamente los trazos de la "hombría" marcado por la rudeza y la omisión del universo emotivo. Esta realidad representa una localidad rural y que pareciera distante de nuestras sociedades más complejas. Sin embargo, encontramos que en contextos como la minería se recrean verdaderos rituales de hombría, a través de un lenguaje soez, bromas y rumores 
(Barrientos et al., 2009). Los hombres tienden a sexualizar los discursos, es decir expresan sapiencia de los significados sexuales.

Frank (2003) para el caso de los strip club, complementa estás ideas y agrega que los clientes no sólo tienen ventajas en términos del capital educativo y social que poseen, sino que, también, en el poder que ejercen los clientes sobre las bailarinas. Esto se observa, igualmente en las interacciones entre clientes y garzonas en la schoperías (Barrientos et al., 2009), ya que los hombres tienden a explicar que estos lugares son relajantes porque son complejos de entretención, sitios que por definición connotan distracción.

Lo anterior justifica la ubicación de las mujeres en un lugar subordinado del sistema social. La sexualización de las relaciones de trabajo expresa la expectativa de que las mujeres deberían actuar como tales (utilizando la seducción, coquetería y femineidad) para relacionarse con los hombres (Tallichet, 1995).

Por consiguiente, la sexualización de los discursos masculinos, la sapiencia de los significados sexuales, el poder que se ejerce en estos espacios de homosociabilidad coadyuva en la configuración de la discriminación. Esta última se refiere a "todo acto de menosprecio, distinción, restricción o preferencia arbitraria hecha -con o sin intención- por persona, grupo o institución sobre la base de una determinada creencia, prejuicio o idea, de que ciertas personas o grupos son inferiores y que atenta contra el derecho a la igualdad" (SEGEGOB, 2004:17). Específicamente, la discriminación de género determina diferencias y relaciones inequitativas entre los géneros masculinos y femeninos, y sostiene la desigualdad que existe entre los beneficios de carácter social y económico que resultan del trabajo de la mujer y la visión social que se tiene de ésta como individuo no productivo. Además, ampara las diferencias con respecto al sexo, roles, posiciones y condiciones entre hombres y mujeres (Quintero, 2007). Por lo tanto, la discriminación como un proceso de diferenciación desigual condiciona la construcción del género y no representa un conjunto de características o roles, sino es el producto de ciertas prácticas sociales que se desarrollan a través de la interacción de los individuos (West y Zimmerman, 1999).

Por tanto, considerando lo expuesto previamente este artículo tiene como objetivo analizar los discursos de los trabajadores mineros del norte de Chile, sobre las mujeres que trabajan en las "schoperías" de Calama, ya que establecemos una relación estrecha entre el lenguaje que utilizan y las identidades masculinas que se recrean en este escenario productivo. La premisa que subyace es que los discursos de los hombres actúan como eje central en el reforzamiento de una masculinidad dominante y en la discriminación de género. 


\section{METODOLOGÍA}

Este estudio se realizó desde un enfoque cualitativo, ya que se concibe la realidad social como subjetiva, múltiple y cambiante (Ruiz, 1996; Valles, 2000; Salinas y Cárdenas, 2008). Se buscó comprender los discursos en un sentido global. Para ello, se realizaron largas jornadas de observación, entrevistas informales y en profundidad a trabajadores-clientes y a dos dueños de locales, estos últimos entregaron información relevante respecto a los trabajadores, las interacciones y dinámica de las "schoperías". Se efectuaron dos grupos de discusión en Calama; en ellos participaron doce trabajadores mineros de la zona. Estás técnicas en su conjunto nos permitieron acceder al discurso individual y colectivo de los participantes en el estudio, de tal manera de conocer las regularidades y variaciones del comportamiento social de los sujetos (Hammersley y Atkinson, 1994; Lipson, 2003).

\subsection{Procedimiento metodológico}

Se analizaron las interacciones de género en cuatro "schoperías" de la ciudad, estos locales fueron escogidos a través de un proceso de observación in situ y de acuerdo a los sugerencias de informantes claves, referidas a que fueran schoperías conocidas en Calama, que tuvieran una antigüedad mínima de cinco años, que asistieran preferentemente trabajadores mineros, y que además nos permitieran ingresar a estos locales, considerando que en el equipo de estudio había una investigadora. Más información respecto a las características de las "schoperías" se puede hallar en Barrientos et al. (2009).

Se realizaron largos períodos de observaciones (50 días en total, preferentemente fines de semana), lo que posibilitó a través de entrevistas informales tomar contacto con los clientes, los propietarios, las garzonas y el público en general. Luego, a través de un informante clave de CODELCO, accedimos a dos grupos de trabajadores que asisten a las "schoperías", de tal manera de realizar los grupos de discusión. Todas las entrevistas fueron grabadas y transcritas exhaustivamente, previa autorización de los participantes. Tuvieron una duración promedio de 90 minutos. Los nombres de los participantes fueron modificados, respetando el principio de confidencialidad de las investigaciones cualitativas. 


\subsection{Análisis}

El análisis de discurso es "un complejo conjunto de actos lingüísticos simultáneos y secuencialmente interrelacionados, actos que se manifiestan a lo largo y ancho de los ámbitos sociales y de la acción" (Wodak, 2003:105). El lenguaje acompaña las prácticas y éstas, a su vez, a gran parte de lo que decimos (Scollon, 2003). El análisis de discurso implicó que nos fijáramos en las palabras como modo de conocer la estructura social, y los procesos de significación y construcción del sentido (Murillo y Mena, 2006). El lenguaje no es un sistema estático y cerrado, ya que la fuerza y la orientación de un enunciado no están determinados exclusivamente por contextos o posiciones previas; por el contrario, un enunciado puede obtener su fuerza, a partir de la ruptura que produce de su contexto, y en estas circunstancias es importante para refutar aquello que ha sedimentado (Butler, 1997). Buscamos hacer un Análisis Crítico del Discurso (en adelante ACD), como una forma de abordar los problemas sociales que se van concatenando en ellos (exclusión, inequidad, entre otros). El ACD es una denominación genérica que se aplica a un planteamiento dedicado a estudiar el habla, el discurso y la comunicación (Van Dijk, 1997; Meyer, 2003). Permite visualizar la relevancia social y política del texto, y muestra una dimensión de significado que sobrepasa el encasillamiento que conlleva un marco situacional concreto (Hennecke, 2004). Diversos estudios críticos del discurso se han focalizado en la discriminación indígena, mapuche, en el caso de Chile (Merino et al., 2008), y a nivel internacional han sido objeto de análisis los discursos políticos, la pobreza, la cultura, el racismo y las construcciones de género (Berardi, 2005; Van Dijk, 1997).

Desde estas propuestas realizamos una codificación del material recopilado en base a la Teoría Fundamentada (Strauss y Corbin, 2002), y con la aplicación que de ella hacen Trinidad y otros (2006). Esta codificación se complementó, además, con la propuesta de análisis discursivo simple de Jäger (2003), ya que este autor se adscribe a esta línea de análisis (Wodak, 2003). Distinguimos en los discursos aquellas figuras retóricas que desvían o tuercen el sentido del texto, ya que son una forma de encubrir la discriminación de género. Entre estas figuras destacamos las metáforas, metonimias y paradojas. Las metáforas son una de las más importantes figuras retóricas, su proceso de desviación (trópico) consiste en establecer una intersección analógica entre dominios diferentes. Las metonimias constituyen un tropo, ya que consiste en la designación de un objeto por el nombre de otro, 
contempla una generalización de una parte por el todo. Y las paradojas son una declaración en apariencia verdadera que conlleva una autocontradicción (Charaudeau y Maingueneau, 2005).

\subsection{Caracterización del contexto de análisis}

De acuerdo a los aportes de Jäger (2003) es importante contextualizar el escenario de análisis. En este sentido, en el norte de Chile, Calama se encuentra ubicada a 215 kilómetros de Antofagasta, con una población aproximada de 143.000 habitantes. En esta ciudad, las schoperías junto a otros locales del rubro constituyen uno de los negocios más rentables. Actualmente, en el centro de la comuna, en un área de 16 cuadras, funcionan 19 schoperías aproximadamente, locales que acogen a los trabajadores mineros. En 1978 se inauguró la primera schopería en la ciudad, nombre que se debe al espumoso líquido derivado de la cerveza. En la década de los 80 se inicia la contratación de mujeres jóvenes y atractivas que provenían de las zonas central y sur del país. La interacción entre hombres y mujeres dio paso a relaciones más erotizadas, adquiriendo relevancia la mujer joven en la rentabilidad del negocio. Con la inclusión de ellas se concentró la entretención masculina, convirtiéndose en la actualidad en la principal forma de esparcimiento de los trabajadores mineros de la zona.

En términos generales, las "schoperías" son espacios de consumo de cerveza. Las garzonas que allí trabajan son en su mayoría solteras con o sin hijos y provienen de Perú, Bolivia y del noreste argentino, o de la zona sur del país, generalmente de la Región del Biobío (Barrientos et al., 2009). Sus edades fluctúan entre los 20 y 35 años de edad. El nivel educacional es variable, algunas tienen la enseñanza media incompleta, otras cuarto año medio rendido, y las menos, son estudiantes universitarias.

\section{RESULTADOS}

A continuación el cuadro sintetiza el proceso de categorización de los datos. En ellos se expresan los distintos ámbitos que aborda la discriminación de género en el discurso. 


\section{Cuadro 1. Categorías centrales.}

\begin{tabular}{|c|c|c|}
\hline Categorías abiertas & Figuras retóricas & Categoría central \\
\hline $\begin{array}{l}\text { 1. El valor económico asociado a las mujeres y } \\
\text { su construcción como objeto de deseo } \\
\text {-Las mujeres son mercancía } \\
\text {-Son objeto de consumo } \\
\text {-Son el producto que se ofrece en la } \\
\text { schopería } \\
\text {-Las mujeres son el modo de atraer gente/ } \\
\text { clientes } \\
\text {-Son como conejo para tener familia (hijos) } \\
\text {-Me tiraron una mujer como perro al hueso } \\
\text {-Entre más vinagre no me sirve, tiene que } \\
\text { ser simpática } \\
\text {-Eran espectaculares, grito y plata (rubias) } \\
\text {-Ella lo tiene pescao ( trabajador) }\end{array}$ & $\begin{array}{l}\text { Metáforas } \\
\text { (mercancía, } \\
\text { objeto, producto) } \\
\text { (conejo, hueso, } \\
\text { vinagre, grito y plata, } \\
\text { pescan a los hombres) }\end{array}$ & Mujer como ganancia. \\
\hline $\begin{array}{l}\text { 2. El control sobre el cuerpo de las mujeres/las } \\
\text { representaciones de lo femenino } \\
\text {-No hay racismo, pero tiene que ser simpática. } \\
\text {-Hay discriminación para elegir a las chicas- } \\
\text { traes una más o menos y otras más feítas. } \\
\text {-Les “mirái” las piernas si son feas no van a } \\
\text { trabajar, su carita flaquita, su buen poto, su } \\
\text { buena teta... } \\
\text {-Las feítas son el boom (favoritas). } \\
\text {-Las cabras que son lindas les cuesta } \\
\text { encontrar novio. } \\
\text {-Puede ser una chiquita, gordita, pero tiene } \\
\text { que tener ángel. } \\
\text {-Tiene que ser una más o menos fea, } \\
\text { entre feíta y más o menos "güeonona” } \\
\text { (curvilíneas). }\end{array}$ & $\begin{array}{l}\text { Paradojas } \\
\text { El cuerpo se escinde, } \\
\text { la cara, las piernas, } \\
\text { el trasero, el pecho } \\
\text { femenino. }\end{array}$ & $\begin{array}{l}\text { El cuerpo como } \\
\text { territorio de dominio. }\end{array}$ \\
\hline $\begin{array}{l}\text { 3. La moral femenina respecto a su honestidad } \\
\text { y a su sexualidad } \\
\text {-Están garzoneando. } \\
\text {-Andan con otros hombres. } \\
\text {-Todas tenían carnet de prostitutas } \\
\text { (garzonas). } \\
\text {-Vino a levantar el negocio (las mujeres). } \\
\text {-Las mejores minas están en la Happy y en } \\
\text { el Vesy. } \\
\text {-Si las echaron es por algo... es un condoro } \\
\text { (falta). } \\
\text {-El } 80 \% \text { de los clientes viene a tirar el } \\
\text { anzuelo por si cae algo. } \\
\text {-Los hombres no se pueden controlar } \\
\text { (deseo sexual). }\end{array}$ & $\begin{array}{l}\text { Metonimia } \\
\text { Robando, tienen } \\
\text { muchas parejas } \\
\text { (promiscuidad), } \\
\text { son fáciles, son } \\
\text { exuberantes, delito. } \\
\text { Pescar mujeres. } \\
\text { Las mujeres provocan. }\end{array}$ & $\begin{array}{l}\text { Las mujeres son } \\
\text { inmorales, no son } \\
\text { confiables como } \\
\text { género. }\end{array}$ \\
\hline
\end{tabular}




\subsection{El valor económico asociado a las mujeres y su construcción como objeto de deseo}

En los discursos analizados, la discriminación de género se expresa en distintas formas, una de ellas se relaciona con la desvaloración del sujeto mujer y la importancia que tienen como ganancia para el negocio de las schoperías. Las garzonas adquieren valor de acuerdo a sus atributos físicos. Se compartimenta su cuerpo satisfaciendo el gusto de los hombres ("El cliente busca de todo -morenas, rubias, trigueñas, entre otras-, cuando 'tení' de todo se llena", propietario de "schopería"). Los dueños de estos locales se fijan en la cara, las piernas, el trasero o el pecho, y son los atributos que se consideran para contratar o no a una garzona.

Las figuras retóricas identificadas en los discursos son una expresión del lenguaje de los hombres que enmarca y legitima la discriminación de género y, específicamente, la dominación masculina. Esta se refiere al fenómeno que subyace en el lenguaje y en la construcción social de la realidad de los sujetos. La desvaloración de lo femenino se expresa en un reduccionismo de ellas en términos del beneficio económico que pueden generar, y las características físicas que tienen para ello. El cuerpo de las mujeres es un territorio en el que se imprime un símbolo, donde la sexualidad es un objeto más de consumo masculino y es el eje articulador de las interacciones entre los clientes y las garzonas. Desde aquí se organiza el negocio: si las mujeres no provocan el interés esperado en los clientes, son rápidamente reemplazadas. Uno de los recursos que se utilizan para destacar los atributos físicos de las garzonas son la vestimenta ajustada, tacos altos y maquillaje. A continuación algunos segmentos de las entrevistas de los propietarios de las "schoperías" y clientes $(\mathrm{EH}, \mathrm{EM})^{5}$ que expresan el valor económicos asociado a la figura femenina:

Ejemplo 1.

"Los cabros miran a las niñas con una cara, como si la estuvieran viendo desnuda".

“Te dicen ven pa' ca cabro hueón ven hacerte hombre con nosotros".

"Su carita flaquita, su buen poto, su buena teta".

"Si tú veí chicas bonitas, la gente va a entrar igual".

\footnotetext{
${ }^{5}$ Siglas EH, EM, etc., corresponden a la identificación de entrevista y la inicial del nombre del informante. Éste fue modificado para resguardar la confidencialidad de los mismos.
} 
Relacionado a lo anterior, en los discursos de clientes se identifican metáforas, las que representan a las mujeres como ganancia, animales o cosas, son productos que se ofrecen, de acuerdo al gusto de los hombres, por ejemplo: "conejos que se reproducen", "carnada que se pesca con anzuelo", "las tiran como huesos que se comen", "minas que se explotan", "mercancía que se vende", "son ácida como el vinagre", entre otras. Estas asociaciones con animales o mercancía refuerzan la condición subalterna e inferior de las mujeres, respecto a la posición de los hombres en este contexto. Inclusive, el uso de expresiones entre los clientes como "es niñita" es usada como descalificación de la masculinidad dominante, se refiere a escasa valentía, fragilidad y cobardía. Los hombres deben distanciarse de las características asociadas a lo femenino, de lo contrario se exponen a ser objeto de burlonas suposiciones, al ridículo, a insultos llenos de hostilidad como "nena" o “maricón" (Cantero, 2003).

Otras expresiones como "iy tu hermana! o “ila vieja tuya!" se usan como descalificativos entre los clientes; en estas circunstancia la figura femenina se tensiona, ya que en el imaginario de los hombres la madre, hermanas, esposa o hijas son representaciones femeninas resguardadas en el espacio privado.

Estas dicotomías disocian la figura femenina, ya que están, por un lado, la esposa, la madre, la hermana, que se deben cuidar y proteger, y "las otras", aquellas que se encuentran en lugares como las schoperías, y que, siendo mujeres, se usan o desechan. Se recrea la dualidad entre mujer-madre / mujer-puta. Algunos ejemplos en las entrevistas (EL, EP, EH, EM, clientes de "schoperías"):

\section{Ejemplo 2. Metáforas}

"Me tiraron una mujer, igual que los perros al tiro el hueso y va a morder".

"Se busca un prototipo de mujer que le guste a los viejos".

"El cliente busca de todo (mujeres), cuando tení de todo se llena... Entre más chicas llegaban, más gente llegaba".

"Se hacen bromas, nos trataban de niñitas".

"Para mí tiene que ser agradable la chica, tiene que atender bien. Mientras más vinagre sea o más se crea el cuento que es linda, no me sirve, tiene que ser simpática”. 
En todas estas expresiones la discriminación se relaciona con el escaso valor de la mujer como sujeto de derecho y la objetivación de su cuerpo.

\subsection{El control sobre el cuerpo de las mujeres/las representaciones de lo femenino}

Los patrones de control del discurso están vinculados estrechamente al poder social. Este se define como una forma de control que un grupo ejerce sobre otro y puede extenderse hasta las acciones y pensamiento del grupo dominado. Si éste beneficia al grupo dominante, también puede ejercerse sobre el texto y el habla. La dominación que se implementa de forma discursiva implica un acceso preferente al texto, que se toma como base o recurso de poder (Van Dijk, 1997; Foucault, 1996).

El control que ejercen los propietarios de las schoperías se manifiesta en los permisos, el cumplimiento de los horarios y en el comportamiento. Las garzonas deben mostrarse sonrientes, de buen ánimo, con una actitud de agrado hacia los clientes, y tener mucho cuidado con quienes se relacionan. Hay normas y reglas que regulan el funcionamiento del local y la forma como organizan su vida; lo central es que todo funcione en favor del negocio. Uno de los propietarios de las schoperías dice (EM):

\section{Ejemplo 3. Control}

"Aquí el horario es sagrado, nadie tiene que estar apurada por irse, porque no tiene ningún apuro, ya que tiene que esperar que llegue el otro turno tranquilita y después se va".

"En la noche todas tienen que irse con la cajera, porque la cajera se lleva la plata, una vez que la cajera está en el colectivo, cada una se va para su lao...".

"Cuando están más tranquilas, pueden atender mejor".

"Se deja que las niñas tomen su trago igual que en los topless, se les paga una comisión".

Evidentemente existen diversos procedimientos de control y delimitación del discurso, que se refieren a la dimensión interna de éste. Otro propietario de "shopería" dice $(\mathrm{EH})$ : 


\section{Ejemplo 4. Control}

"Todas tienen que sentirse libre, usted haga lo que quiera, pero si se desordena o se pone a pololear con un hueón traficante, un hueón ordinario yo la voy a mandar a la cresta, porque el negocio es mío".

En los discursos de los hombres se destacan símbolos e imágenes de lo femenino. Estas son representación perceptible de una realidad, con rasgos asociados y compartidos por un grupo o comunidad lingüística. Al respecto es notoria la discriminación que aquí se recrea, ya que las representaciones del ser mujer se relacionan con las expectativas que tienen los hombres sobre cómo deben ser ellas para este negocio. Los atributos físicos son el eje articulador. Se utilizan formulaciones paradójicas como: "pero si es fea no va a trabajar"; "puede ser gorda, pero tiene que tener simpatía"; "puede ser chiquita, pero tener ángel”. La discriminación alude al cuerpo femenino y como territorio de dominio se escinde, y adquiere un valor en sí mismo, como lo veíamos también presente en la forma de metáforas. En las paradojas hay una contradicción que resulta de una deducción correcta a partir de premisas incongruentes; son órdenes que incluyen información contradictoria. De los discursos se extrae un imaginario de belleza femenina asociada al color de la piel y el cabello. Ojalá todas fuesen blancas, rubias, delgadas, pero que tengan curvas. Lo paradojal es que cuando las garzonas son buenas mozas, producen inseguridad en los clientes, temen ser rechazados. En las entrevistas hay expresiones dicotómicas, tanto propietarios como clientes afirman (EH, EL, EM):

Ejemplo 5. Paradojas

"La vieja de la agencia (de empleo) te dice tengo una chica espectacular, linda preciosa y tú agarray papa hueón... y tú vay al terminal a buscarla y te dan puras ganas de comprarle los pasajes y mandarla de vuelta".

"La mayoría de las cabras que son lindas les cuesta encontrar novio, porque el hueón tiene temor al rechazo".

"Mientras más vinagre o más se crea el cuento que es linda, no me sirve, tiene que ser simpática".

"No hay racismo, pero tienen que ser simpáticas".

“Tiene que ser una más o menos fea, entre feíta y más o menos "güenona”. 
Las formas de expresión más usadas son ambiguas, sin embargo anidan una discriminación que pareciera imperceptible y naturalizada, de tal modo que no produce mayor asombro en los sujetos. Se utilizan recursos lingüísticos indirectos, donde se tuerce el sentido de la palabra. El uso de metáforas, metonimias y paradojas son una muestra de aquello, ya que los desvíos o torsiones argumentativas del lenguaje transversaliza el habla de los trabajadores.

\subsection{La moral femenina respecto a su sexualidad y a su honestidad}

Las calificaciones de los hombres sobre las mujeres tienden a ser formulaciones generales, "las mujeres roban", "se emparejan", "son fáciles", "se prostituyen", "son las mejores minas", "son exuberantes", "tienen hijos como conejos" y "están detrás de la plata de los trabajadores". Estas expresiones se refieren a la frágil moralidad de las mujeres, y aluden al ejercicio de su sexualidad, pues están al servicio de los hombres o a que ellas no son honestas, "andan con uno y con otro", "son poco confiables puesto que la mayoría roba" y "les interesa el dinero del trabajador". En estas expresiones se identifican la discriminación sistemática que encierra el discurso masculino, el escaso valor que se le confiere al sujeto mujer, el valor dual de la mujer-madre versus la mujer-"puta" y la ligereza con que se evalúa o interpreta el comportamiento femenino. Este lenguaje se refuerza bajo el amparo de la dinámica de interacción y complicidad que se genera entre los hombres. Estas generalizaciones en el discurso masculino se pueden identificar retóricamente como metonimias, ya que es una manera de señalar una parte de la realidad como representación del todo (Charaudeau y Maingueneau, 2005). Estas expresiones generales sobre las mujeres se relacionan, principalmente, con aspectos éticos que cuestionan la honestidad y la moralidad de ellas. Se construyen, de este modo, prejuicios que facilitan la discriminación hacia las garzonas. Algunos segmentos de entrevistas que grafican estas generalizaciones. EM (propietario de schopería) y EP (cliente) dicen:

Ejemplo 6. Metonimias

"Mi mamá lleva todo el control para evitar el robo hormiga...".
"Las chicas que trabajaban todas tenían carnet de prostitutas".
"Muchas mujeres ven al compadre de Codelco con esos ojos (dinero)" 
Las mujeres dicen: "mientras encuentre unos hueones para que me alimenten..."

\section{CONCLUSIONES}

La discriminación se articula en torno a tres categorías centrales: a) el valor económico asociado a las mujeres y su construcción como objeto de deseo; b) el control sobre el cuerpo de las mujeres/las representaciones de lo femenino, y c) la moral femenina respecto a su sexualidad y honestidad.

Estas expresiones de la discriminación que son recreadas en estos espacios de esparcimiento, actúan como refuerzo de una identidad masculina dominante, que se sostiene en los roles o representaciones que se le adscriben al ser hombre y/o mujer. En las relaciones de género que se construyen en estos locales, el ejercicio del poder es asimétrico, de modo que los intereses de las mujeres están subordinados a los de los hombres, lo que se refleja en las interacciones que se construyen. Es decir, se mantiene un orden de género dominante (hombre activo - mujer pasiva) y un conjunto de representaciones sociales que sustenta un enclave cultural de subordinación, control y discriminación (garzonas son mujeres fáciles, que ejercen el comercio sexual).

La discriminación y la identidad masculina dominante encuentran en los discursos de los hombres formas de expresión caracterizadas por un lenguaje indirecto. Ellos utilizan figuras retóricas que favorecen representaciones sociales difíciles de modificar. Las metáforas se centran en las características asociadas a la sexualidad de las mujeres y la carga valorativa que se relaciona a ella, esto último directamente asociado a la relevancia económica que se le asigna al cuerpo femenino, como mercancía y producto de consumo, se realza la juventud, la belleza, la simpatía como atributos que deben acompañar a las mujeres que atienden en las "schoperías", ya que estas propiedades hacen rentable y atractivo el lucrativo negocio. Las metonimias o generalizaciones que se refieren a las garzonas tienen principalmente una connotación negativa que atañe a su dignidad como sujeto.

Por consiguiente, es importante develar la vigencia de esta identidad masculina dominante en este contexto productivo, pues se trata de un escenario vinculado a una de las actividades económicas más importantes del país, por ende, el impacto social y cultural de esta realidad es significativo, ya que los hallazgos de esta investigación se encuentran concatenados a una realidad mayor de inequidad, asimetrías sociales y de género, y a profundos 
desequilibrios entre los avances económicos de la región, la calidad de vida y las transformaciones culturales de la zona (Cademártori, 2009).

Podemos afirmar que las condiciones laborales y culturales de la minería contribuyen a la vigencia de una identidad masculina dominante. Las prácticas organizacionales de la actividad mantienen este orden, basado en esta identidad estereotipada, donde la rudeza, resistencia y fuerza son valores centrales. La discriminación de género es un fenómeno que se sostiene en este contexto.

Algunas de las limitaciones que ha presentado este estudio se refieren a la complejidad que implica analizar la discriminación, por la diversidad de factores asociados a esta problemática, por lo tanto se requieren más investigaciones en el área de tal manera de avanzar en la profundidad del análisis, por ejemplo explorando otros discursos (prensa, medios, empresas). Además, es necesario desarrollar iniciativas que apunten a iniciar la modificación de esta realidad, ya que la minería, como la principal actividad productiva en la zona, requiere desarrollar políticas de carácter transversal que apunten a discutir el orden de género vigente, potenciando la deconstrucción de los discursos sobre las mujeres como sujeto femenino, en tanto disvalor. El desarrollo regional debe procurar un crecimiento más equilibrado de los distintos ámbitos de la vida social. Así, los aspectos culturales en este contexto son centrales, allí los discursos de género, los roles que desempeñan mujeres y hombres en la sociedad constituyen un eslabón fundamental para cimentar la calidad de vida de los habitantes de la zona, acorde a los valores democráticos e igualitarios en boga en la cultura chilena.

\section{REFERENCIAS}

Aylwin, M.; Bascuñán, C.; Correa, S.; Gazmuri, C.; Serrano, S. y Tagle, M. (1998). Chile en el siglo XX. Octava edición (6ta. en Planeta). Santiago de Chile: Planeta.

Barrientos, J.; Salinas, P.; Varas, P. y Meza, P. (2009). “Minería, género y cultura. Una aproximación etnográfica a espacios de esparcimiento y diversión masculina en el norte de Chile". AIBR - Revista de Antropología Iberoamericana, 4(3), 385-408.

Berardi, L. (2005). Análisis crítico del discurso. Perspectivas latinoamericanas. Santiago de Chile: Frasis Editores.

Bourdieu, P. (2007). La dominación masculina. España: Anagrama Colección. Butler, J. (1997). Lenguaje, poder e identidad. España: Editorial Síntesis.

Cantero, P. (2003). "Hombrear. Modos de aprender a ser hombre". En. José María Valcuende del Río, Juan Blanco López (editores), Hombres. La cons- 
trucción cultural de las masculinidades. Madrid: Talasa Ediciones S.L Serie Arcoíris, 53-65.

Carabí, A. y Armengol, J. (2008). La masculinidad a debate. Barcelona: Icaria Mujeres y Cultura,

Careaga, G. y Cruz, S. (2006). Debates sobre masculinidad. Poder, desarrollo, políticas públicas y ciudadanía. México: Universidad Nacional Autónoma de México.

Cademartori, J. (2009). El desarrollo económico y social de la Región de Antofagasta (Chile). Historia y perspectiva. Antofagasta: Universidad Católica del Norte.

Carmagnani, M. (2006). El salario minero en Chile colonial, su desarrollo en una sociedad provincial: El norte chico 1690-1800. Santiago de Chile: Dirección de Bibliotecas, Archivos y Museos.

Charaudeau, P. y Maingueneau, D. (2005). Diccionario de análisis del discurso. Madrid: Editores Amorrortu.

Connell, R. (2003). Masculinidades. México: PUEG. Universidad Nacional Autónoma de México. Programa Universitario de Estudios de Género.

— (2006). “Desarrollo, globalización y masculinidades”. En G. Careaga y S. Cruz Sierra (Eds.), Debates sobre masculinidades. Poder, desarrollo, politicas públicas y ciudadanía. México: Universidad Autónoma de México, 185-210.

Cortés, J. M. (2004). Hombres de mármol. Códigos de representación y estrategias de poder de la masculinidad. Barcelona: Editorial Egales.

Dunbar Moodie, T. (1994). Going for gold: men, mines and migration. Johannesburg: Witwatersrand University Press.

Foucault, M. (1996). El orden del discurso. Madrid: Las Ediciones de La Piqueta.

Frank, K. (2003). Just trying to relax: masculinity, masculinizing practices, and strip club regulars. Journal of Sex Research, 48 (1), 61-75.

Fuller, N. (1998). "Reflexiones sobre el machismo en América Latina”. En. T. Valdés \& J. Olavarría. (Eds.), Masculinidades y equidad de género en América Latina. Santiago: FLACSO-UNFPA, 258-266.

GIM (Grupo Iniciativa Mujeres) (2002). El nuevo contrato social: Balance de una década de democracia en Chile. Santiago: Cedem, Cem, Corporación de La Morada, CORSAPS, Domos, FLACSO, Fundación Instituto de la Mujer, Isis Internacional, MEMCH Y PROSAM.

Hammersley, M. y Atkinson, P. (1994). Etnografía. Métodos de investigación. Barcelona: Paidós Básica.

Hennecke, A. (2004). Diskurs als Fluss von Wissen duch die Zeit. Reflexionen über das Verhältnis zwischen Diskurs und Wirklichkeit. Argentina: Comunicarte editorial.

Jäger, S. (2003). "Discursos y conocimiento: aspectos teóricos y metodológicos de la crítica del discurso y del análisis de dispositivos”. En Métodos de análisis crítico del discurso, Ruth Wodak y Michael Meyer (compiladores). España: Gedisa, 61-100.

Jardim, D. (1995). "Performances, reprodução e produção dos corpos mascu- 
linos". En O. Leal (editora), Corpo e significado Rio Grande do Sul, Brasil: Universidade Federal do Rio Grande do Sul, 93-207.

Klubock, T. (1995). "Hombres y mujeres en el teniente, la construcción de género y clase en la minería chilena del cobre 1904-1951”. En Godoy, L. [et al.], Disciplina y desacato. Construcción de identidad en Chile, siglos XIX y $X X$. Santiago de Chile: Coedición SUR/CEDEM, 110-123.

Lamas, M. (2002). Cuerpo: Diferencia sexual y género. México: Taurus Pensamiento. Lipson, J. (2003). “Asuntos éticos en la etnografía”. En. J. M. Morse (editora), Asuntos críticos en los métodos de investigación cualitativa. Contus, Colombia: Facultad de Enfermería de la Universidad de Antioquia, 389-415.

Macheke, C. y Campbell, C. (1998). "Perceptions of HIV/AIDS on a Johannesburg gold mine”. South African Journal of Psychology, 28(3), 146-154.

Martínez, G. (2007). "La construcción imaginaria de la sexualidad y la violencia masculina”. En. R. Montesinos (coord.), Perfiles de la masculinidad, México: Universidad Autónoma Metropolitana, 47-73.

Merino, M. E.; Quilaqueo, D. y Saiz, J. L. (2008). "Una tipología del discurso de discriminación percibida en mapuches de Chile”. Revista Signos, 41 (67), 279-297.

Meyer, M. (2003). "Entre la teoría, el método y las políticas: la ubicación de los enfoques relacionados con el ACD”. En Ruth Wodak y Michael Meyer, Métodos de análisis crítico del discurso. Madrid: Gedisa, 35-59.

Montecino, S. (1998). Análisis de impacto psicosocial, sistema de trabajo por turnos en la unidad familiar. Antofagasta: SERNAM. [En línea]. Disponible en: http://www.areaminera.com/contenidos/entrevistas/entrevistas/21.act [Consultado 25 de octubre, 2009].

Montesinos, R. (2002). Las rutas de la masculinidad. Ensayos sobre el cambio cultural y el mundo moderno. España: Gedisa.

— (2007). Perfiles de la masculinidad. México: Universidad Autónoma Metropolitana.

Murillo, S. y Mena, L. (2006). Detectives y camaleones: el grupo de discusión. Una propuesta para la investigación cualitativa. Madrid: Talasa Ediciones.

Olavarría, J. (2000). "De la identidad a la política: Masculinidades y políticas públicas: Auge y ocaso de la familia nuclear patriarcales en el siglo XX”. En J. Olavarría y R. Parrini (eds.), Hombres: Identidad/es y violencia. Encuentro de Estudios de Masculinidades: Identidades, cuerpos, violencia y políticas públicas. FLACSO- Chile, Santiago: Universidad Academia de Humanismo Cristiano/Red de Masculinidades, 11-28.

- (2006). "Hombres e identidad de género. Algunos elementos sobre los recursos de poder y violencia masculina". En: Gloria Careaga y Salvador Cruz (coords.), Debates sobre masculinidades. Poder, desarrollo, políticas públicas y ciudadanía. México: Universidad Autónoma de México, 115 -130.

Quintero, Á. (2007). Diccionario especializado en familia y género. Argentina: Editorial Hvmanitas de Editorial y Distribuidora Lumen.

Ruitenbeek, H. (1967). El mito del machismo. Buenos Aires: Paidós. 
Ruiz, J. (1996). Ignacio. Metodología de la investigación cualitativa. Bilbao: Universidad de Deusto.

Ruiz, O.; Solano, S. y Zapata, C. (1998). "Redes de mujeres pobladoras de la comuna de San Joaquín. Participación y ciudadanía emergente”. Proposiciones No 28. Santiago: Sur Ediciones.

Salazar, G. y Pinto, J. (1999). Historia contemporánea de Chile II, actores, identidad y movimiento. Santiago de Chile: Lom.

Salinas, P. (2004). "Ciudadanía y género. Mujeres y liderazgo en las provincias de Antofagasta, El Loa y Tocopilla”, Revista Electrónica de Trabajo Social No 5 (Universidad de Concepción) [En línea]. Disponible en: http://www2. udec.cl/ ssrevi/numero5/ [Consultado 10 de diciembre, 2009].

Salinas, P. (2007). "Los discursos masculinos como dispositivos de control y tensión en la configuración del liderazgo y empoderamiento femenino". Revista de Estudios Feministas del Centro de Filosofía y Ciencias Humanas, 15(3), 541-562.

Salinas, P. y Arancibia, S. (2006). "Discursos masculinos sobre el poder de las mujeres en Chile, Sujetos y subjetividades”. Última Década (CIDPA), 25, 65-90.

Salinas, P. y Cárdenas, M. (2008). Métodos de investigación social. Una aproximación a las estrategias cuantitativas y cualitativas. Antofagasta: Universidad Católica de Antofagasta.

SEGEGOG (Secretaría General de Gobierno) (2004). Agenda Participación Ciudadana. División Organizaciones Sociales. [En línea]. Disponible en: www.goreatacama.mix.cl/sysdataweb/notas/ficheros/Copiapo\%5B1\%5D. ppt - [Consultado 15 de octubre, 2009].

Scollon, R. (2003). "Acción y texto: Para una comprensión conjunta del lugar del texto en la (inter)acción social, el análisis mediato del discurso y el problema de la acción social”. En Wodak Ruth y Meyer Michael (coords.), Métodos de análisis crítico del discurso. España: Gedisa Editorial, 205-267.

Seidler, V. (2006). Masculinidades. Culturas globales y vidas intimas. España: Montesinos Ensayo.

Strauss, A. y Corbin, J. (2002). Bases de la investigación cualitativa. Técnicas y procedimientos para desarrollar la teoría fundamentada. Medellín: Editorial Universidad de Antioquia.

Tallichet, S. (1995). "Gendered relations in the mines and the division of labor underground”. Gender \& Society, 9(6), 697-711.

Todaro, R.; Mauro, A. y Yáñez S. (2000). “Chile: la calidad del empleo. Un análisis de género”. En María Elena Valenzuela y Gerhard Reinecke (eds.), ¿Más y mejores empleos para las mujeres? La experiencia de los países del MERCOSUR y Chile. Santiago de Chile: OIT, 193-265.

Trautner, M. (2005). "Doing gender, doing class: the perfomance of sexuality in exotic dance club”. Gender \& Society, 19(6), 771-788.

Trinidad, A.; Carrero, V. y Soriano, R. M. (2006). Teoría fundamentada “Grounded Theory”. La construcción de la teoría a través del análisis interpretacional. Madrid: Centro de Investigaciones Sociológicas. 
Valcuende, J.; Blanco, J. (2003). Hombres. La construcción cultural de las masculinidades. Madrid: Talasa ediciones. Serie Arcoiris.

Valdés, T. (coord.) (2001). El indice de Compromiso Cumplido-ICC. Una estrategia para el control ciudadano de equidad de género. Santiago: FLACSO. [En línea]. Disponible en: http://cronopio.flacso.cl/fondo/pub/digitalfree/2001/ libro/002702.pdf [Consultado 15 de noviembre, 2009].

Valdés, T. y Olavarría, J. (eds.) (1997). Masculinidad/es poder y crisis. Santiago, Chile: ISIS Internacional/FLACSO.

Valdés, T. y Weinstein, M. (1993). Mujeres que sueñan: las organizaciones de pobladoras 1973-1989. Santiago: FLACSO.

Valles, M. (2000). Técnicas cualitativas de investigación social. Reflexiones metodológicas y práctica profesional. Madrid: Síntesis.

Van Dijk, T. (1997). Racismo y análisis crítico de los medios. Barcelona: Editorial Paidós.

Vergara, A. (2004). "Conflicto y modernización en la Gran Minería del Cobre (1950-1970)". Revista de Historia del Instituto de Historia de la Pontificia Universidad Católica de Chile, 37(2), 419-436.

_ (2007). "Ciudades privadas. La vida de los trabajadores del cobre”. En R. Sagredo, C. Gazmuri (eds.), Historia de la vida privada en Chile. El Chile contemporáneo. De 1925 a nuestros días. Santiago de Chile: Taurus-Aguilar, 84-103.

Viveros, M. (2001). Contemporary Latin American Perspectives on Masculinity. Men and Masculinities, 3(3), 237-260.

West, C. y Zimmerman, D. (1999). "Haciendo género”. En. M. Navarro, C. \& R. Stimpson (compiladoras), Sexualidad, género y roles sexuales. México: Fondo de Cultura Económica, 109-143.

Wodak, R. (2003). "De qué trata el análisis crítico del discurso (ACD). Resumen de su historia, sus conceptos fundamentales y sus desarrollos”. En Wodak, Ruth y Meyer, Michel. Métodos de análisis crítico del discurso. España: Gedisa, 17-34.

Zapata, F. (2002). "Los mineros como actores sociales y políticos en Bolivia, Chile y Perú durante el siglo XX”. Estudios Atacameños No 22, 91-103. [En línea]. Disponible en: http://www.scielo.cl/scielo.php?pid=S0718-1043200 2002200006\&script=sci_arttext [Consultado 15 de octubre, 2009). 